\title{
Teaching with emerging technologies in a STEM university math class
}

\section{Chiara Andrà $^{1}$, Giulia Bernardi ${ }^{2}$, Domenico Brunetto ${ }^{2}$}

${ }^{1}$ Department of Science, Università del Piemonte Orientale, Italy, ${ }^{2}$ Department of Mathematics, Politecnico di Milano, Italy.

\begin{abstract}
The aim of the research presented in this work is to investigate how innovative teaching formats, based on student-centred activities, may help first year university students to deal with the difficulties in the transition from the mathematics they are used to in high school, to the one they meet at university, which requires a significant shift to conceptual understanding, especially in Calculus courses. As part of this overarching goal, this presentation investigates the case of Taylor series, a topic that is taught in all calculus courses at university. This work shows the efficacy of a blended learning approach, highlighting the main difficulties concerning the deep understanding of functions by students. We discuss possible limitations, and we provide suggestions for best practices in university math classes.
\end{abstract}

Keywords: Blended learning; Calculus; First-year university mathematics; Formative assessment; Socrative; Taylor series. 


\section{Introduction}

Calculus is one of the fundamental courses in all STEM programs, but even Engineering students deal with difficulties in mathematics, especially at the beginning of their studies (Gómez-Chacón, Griese, Rösken-Winter, \& Gónzalez-Guillén, 2015). Many researches in Mathematics Education point out that difficulties are rooted in the differences between high school and university (Gueudet, 2008). Tall (2004) suggests to consider the transition as an "abstraction shock", since university mathematics adds a formal world to the mathematics encountered at school, and Clark and Lovric (2008) add that students live a leap from a focus on the procedural aspects to conceptual understanding that university mathematics entails.

Within calculus syllabus, topics like series, power series and Taylor series appear at surface level as procedural, but they require a deep conceptual understanding of function image, limits, infinite sum, the convergence radius, and approximation. The dual nature of the topic poses specific challenges to the students. The research problematique addressed in this work regards the way it is possible to support the move from procedural to conceptual approaches to mathematics in first-year calculus courses. A specific focus is on Taylor series, which requires a robust conceptual thinking without giving up procedural abilities.

In order to enhance conceptual learning, many scholars (e.g., Gamer and Gamer, 2001) have proven that student-directed learning promotes it more effectively if compared to traditional teacher-directed instruction. Blended learning formats are a way to encourage studentdirected learning, both because the students are left alone, at home, in the process of meaningmaking, and because in class they are involved in group work and discussions.

On the basis of these premises, the presentation unfolds as follows. Firstly, we recall the relevant aspects of Taylor series. Then, we describe how we designed and carried out a student-centred activity in a first-year calculus course for Building Engineering students at Politecnico di Milano. Finally, we briefly discuss the main findings.

\section{Mathematical content: Taylor series expansion}

The Taylor series expansion of a function $f(x)$ centred at a given value, $x=x_{0}$, is a power series in which each coefficient is related to a derivative of $f(x)$ with respect to $x$. Some common uses of Taylor series include numerical computations, evaluations of definite integrals and/or indeterminate limits, and approximations. The series is often truncated by choosing a finite upper limit for the summation on the basis of a certain criterion. The Maclaurin series is a special case, where $x_{0}$ is set to 0 .

According to Smith, Thompson, and Mountcastle (2013), a small number of studies in Mathematics Education investigate students' understanding and use of Taylor series and in general the topic is not the main focus of the research, but only part of a broader project. 
However, a dominant theme emerging from many articles is the difficulty of synthesising many previously learned calculus concepts to generate a robust understanding of Taylor series (see Smith, Thompson \& Mountcastle, 2013). A specific focus is on graphical understanding of Taylor series. Interestingly, Habre (2009) found that visual reasoning of Taylor series convergence may be possible even for students with poor mathematical backgrounds and who may not be able to reason about convergence analytically. Hence, such an approach to Taylor series seems relevant for students who struggle with the conceptual approach of mathematics in STEM university course.

In the context of a physics course, Smith, Thompson, and Mountcastle (2013) investigate how students recall the relationship between the algebraic forms of Taylor series and the graph of the function. We share the same cognitive focus, but the context of our research is a first-year mathematics course, and our specific interest is on investigating how students create an understanding of this relationship the first time they encounter it.

\section{Research methods}

\subsection{Data collection}

In this presentation, we consider the data collected during a teaching activity on Taylor series. On the first day of the Calculus course, the students filled in an anonymous questionnaire, which allowed us to know their gender and the high school of provenience. Other three questions investigate their attitudes towards mathematics. The information provided by this questionnaire, which is shown in the next section, informed the design of the blended learning activity, which consisted of a homework assigned via email, and a lecture, which started with an activity with Socrative, an educational platform which allows delivering instant feedback poll in a large classroom. The results of our research regards the lecture.

\subsection{Description of the sample}

The students involved were 126 , of which $54 \%$ males, and $11 \%$ had a weak mathematical background according to their high school of provenience, as shown in Table 1. 
Table 1. Description of the sample.

LS=science and mathematics majors, $\mathrm{TE}=$ applied mathematics, $\mathrm{HU}=$ humanities majors.

\begin{tabular}{cccccc}
\hline \multicolumn{2}{c}{ Gender } & \multicolumn{4}{c}{ High School type } \\
\hline Male & Female & LS & TE & HU & Other \\
& & & & & \\
$54 \%$ & $46 \%$ & $70.6 \%$ & $18.3 \%$ & $4.8 \%$ & $6.3 \%$ \\
\hline \multicolumn{5}{c}{ Source: by authors. } \\
\hline
\end{tabular}

The first question on students' attitudes towards mathematics concerned how to deal with math problems - What do you do when you deal with a difficult math problem?- , the second question investigated students' attitudes towards mathematical problems (Figure 1), and the last question was related to the use of online resources to study mathematics (Figure 2).

The answers given by students to question 1 reveal that when they deal with a difficult mathematical problem the majority of them (38.1\%) uses to refer back to a simpler problem, however many of them declare they use online resources, such as the web (23\%) and social media (20.6\%), while the $15.9 \%$ of students ask the teacher. Only $2.4 \%$ of them declares that they give up. From the second question, it emerges that students assign a procedural value to a mathematical problem, but they also think that a similar problem can be useful for deepening their mathematical knowledge (Figure 1). Finally, the answers to the last question show that forums and online videos are used for studying math (Figure 2).

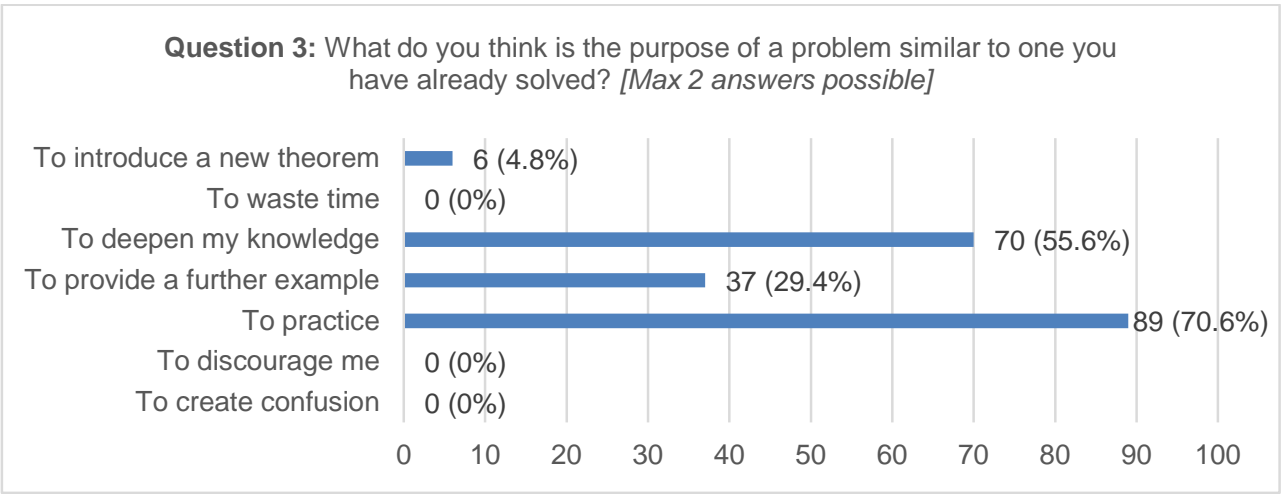

Figure 1. Students' answers to question 2. Source: authors 


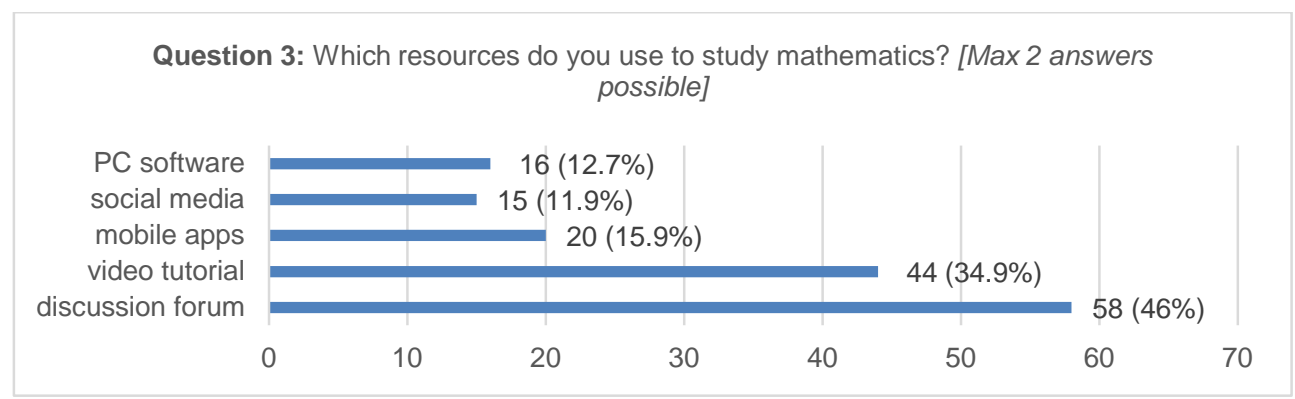

Figure 2. Students' answers to the question 3. Source: authors

From the questionnaire, we can infer that students involved in the project were confident with online resources and had a procedural view of mathematics.

\subsection{Lesson design}

In designing our lesson, we exploited the students' good acquaintance with web resources, but we also wanted to provoke a change in their approach to mathematics. To do so, we invited the students to engage in homework following these steps:

1. watch the video "Taylor Series" ${ }^{1}$ as an introduction, which focuses explicitly on the relationship between algebraic and graphical representations of Taylor series;

2. read and study an online page ${ }^{2}$, as a way to synthesise the previous video and to fix the ideas on the algebraic form of Taylor series;

3. given an example of a function, explore the graphical meaning of its Taylor series expansion through a Geogebra applet ${ }^{3}$.

The goal of this activity is not only to introduce the concepts and to understand the relationship between the graphical and the algebraic approaches, but also to get sense of approximation of a function by means of Taylor series. An email was sent five days before the lecture and asked the students to work at home following the above steps. They had been informed that their work would have been the starting point of the lecture.

\subsection{Geogebra activity as homework}

The applet was designed using Geogebra online applet. The learning environment provides an interactive graph (shown in Figure 3), where students can explore the Taylor series providing the function and the centred point as inputs; the order $n$ can be changed through a

\footnotetext{
${ }^{1}$ The link to the english video is https://www.youtube.com/watch?v=3d6DsjIBzJ4

2 The link to the Italian discussion forum is https://www.youmath.it/lezioni/analisi-matematica/derivate/537-come-sviluppare-unafunzione-in-serie-di-taylor.html

3 The link to the Geogebra applet [Italian language] is https://www.geogebra.org/m/cbcz32vz
} 
slider, and it is possible to visualise all the terms of the Taylor series. With the purpose of prompting students' thinking about the goal of the activity, namely the algebraic and graphical relationship and the function approximation, some suggestions are provided: 1) What happens when you increase the order? 2) The Taylor series allows you to approximate functions with polynomials. Do you agree? Why? 3) What happens if the function $f(x)$ is a polynomial? Make conjectures and try! 4) Consider the Taylor series of $f(x)=e^{x}$ at $x=1$. What is the maximum value of the error (namely the distance between the two graphs) within the interval $[0,2]$ varying the order $n$ of the series? And within the interval $[-2,2]$ ?

The goal of the homework was to prime the students to the concepts related to Taylor series and the questions were given in order to stimulate their reflection and elaboration of both mathematical theory and their exploration of the given example. The lecture started with a Socrative activity that, assigning similar questions and another example, serves multiple purposes: for example, fixing the student's ideas and letting misinterpretations and mistakes emerge.

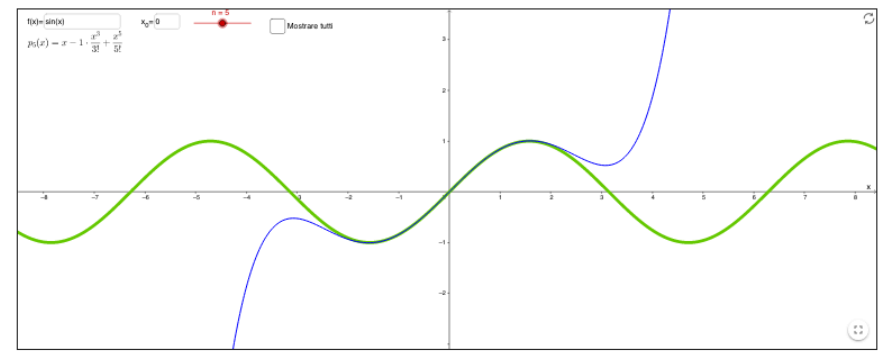

Figure 3. The Geogebra applet designed for the students' homework. Source: by authors from https://www.geogebra.org/m/cbcz32vz

\subsection{Socrative activity in class}

At the beginning of the lecture, the students were engaged in answering seven questions on Socrative reported in Table 2: questions 1 and 2 are multiple choice, questions 3 and 4 require to give a short answer, while the remaining three ones are of type true/false regarding the figure in question. Correct answers are given in brackets, for the reader's sake. 
Table 2. Questions delivered through Socrative during the lecture.

1. The MacLaurin series of a function is...

a. the Taylor series centred at $x=0$ [correct]

b. the Taylor series up to order 10

c. the Taylor series up to order 10 centred at $x=0$

d. the function series with polynomials

2. The Taylor series of a function allows you to...

a. compute the function's derivatives

b. approximate the function with polynomials [correct]

c. approximate the function as sum of its derivatives

d. compute the function's value at certain points

3. Which is the Taylor series up to order 3 at $x=0$ of $f(x)=x^{3}+3 x^{2}+10 x-1$ ? [itself]

4. Which is the Taylor series up to order 5 at $x=10$ of $f(x)=x^{3}+3 x^{2}+10 x-1$ ? [itself]
5. The graph reports the Taylor series up to order 3 at $x=1$ of $f(x)=\cos \left(x^{2}\right)$. [true]

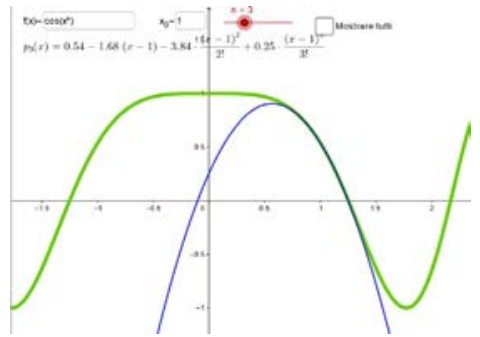

6. The Taylor series up to order 3 at $x=1$ of $f(x)=\cos \left(x^{2}\right)$ is a good approximation of $f(x)$ within the interval $[0,1]$. [false]

7. The Taylor series up to order 3 at $x=1$ of $f(x)=\cos \left(x^{2}\right)$ approximates the function with an error below 1 with the interval $[0,1]$. [true]

\section{Results from the Socrative activity}

The number of students who participated in the Socrative activity is 98 . The average number of correct answers is 5.45 over seven questions. Table 3 reports the percentages of correct answers to each question.

Table 3. Percentage of correct answers for each question

\begin{tabular}{ccccccc}
\hline Q1 & Q2 & Q3 & Q4 & Q5 & Q6 & Q7 \\
\hline $80.6 \%$ & $95.9 \%$ & $81.6 \%$ & $42.9 \%$ & $73.5 \%$ & $87.8 \%$ & $82.7 \%$ \\
\hline
\end{tabular}

The question Q1 is a mere verification of the definition provided in the online report, and the high percentage of correct answer shows that the students did the homework. We can notice that the huge majority of students replied correctly to question Q2. We can take this result as an indicator that the students watched the video assigned at home, but also that they understood the definition of the mathematical concept. The relatively high percentage of correct answers to question Q5 show that students explored at home the applet attentively. Questions Q6 and Q7 require to observe the graph and to comment on the approximation. From the answers to these questions we can infer that the activity with the applet at home helped them to figure out the concept of approximation of a given function by means of its Taylor series. The question Q3 and Q4 concern the property that the Taylor series of a polynomial function is the function itself, we highlight that some suggestions in the 
homework were focused on this property. The different percentage of right answers to the two questions shed light on the difficulties of students with this topic. Looking at the answers in details, we can argue that the majority of students were not using the property but were explicitly computing the terms of the Taylor series of the polynomial. For instance, in the samples selected in Table 3 it is evident that students either computed the expansion of the polynomial function or tried to compute it. We stress that, even if the answers to Q3 given by the three students are formally correct, as well as the student 3's answer to Q4, they show that the students were not grasping the meaning of the activity prompted by question Q2.

Table 4. Example of answers to questions Q3 and Q4

\begin{tabular}{ccc}
\hline Student & Answers to Q3 & Answers to Q4 \\
\hline S1 & $\mathrm{F}(\mathrm{x})=-1+10 \mathrm{x}+3 \mathrm{x}^{\wedge} 2+\mathrm{x}^{\wedge} 3$ & I need more time \\
$\mathrm{S} 2$ & $\mathrm{f}(\mathrm{x})=-1+10 \mathrm{x}+6 \mathrm{x}^{\wedge} 2 / 2 !+6 \mathrm{x}^{\wedge} 3 / 3 !$ & $\mathrm{f}(\mathrm{x})=-1+10(\mathrm{x}-10)+6(\mathrm{x}-10)^{\wedge} 2 / 2 !+6(\mathrm{x}-10)^{\wedge} 3 / 3 !$ \\
$\mathrm{S} 3$ & $\mathrm{f}(\mathrm{x})=\mathrm{x}^{\wedge} 3+3 \mathrm{x}^{\wedge} 2+10 \mathrm{x}-1$ & $\mathrm{f}(\mathrm{x})=1399+370(\mathrm{x}-10)+33(\mathrm{x}-10)^{\wedge} 2+(\mathrm{x}-10)^{\wedge} 3$ \\
\hline
\end{tabular}

Moreover, the tutors who were present during the lecture reported that this point was the most discussed one with students. To support the property behind these questions, tutors computed the Taylor series using the definition like Student 3, but they simplified the result to explicitly obtain the given polynomial function. This proof, together with the use of the Geogebra applet, were the key elements to make the students aware of the meaning of a Taylor series and the relationship between the algebraic and the graphical representations.

\section{Conclusions}

To recall, the focus of our research is on how to support the students' conceptual understanding of Taylor series the first time they encounter this topic. In particular, we are interested in developing a deep understanding of the relationship between its algebraic and graphical representations. To this end, the results of our investigation reveal that the video and the text, respectively watched and read at home, reached the goal of supporting students' learning of Taylor series (and MacLaurin series). Paired with the homework activity with the Geogebra applet, the video and the text were also effective in enhancing the application of the concept to examples of functions, in a way that is manipulative, explorative and questionbased. In fact, the student were successful in answering questions about other, new functions given in class. In this way, emergent technologies sustain the students' deep understanding of a challenging topic. True, a limitation of our findings is that the success of the activity depends on the students' willingness to engage in homework, which is not always the case (as it is outlined by researchers on Flipped Classroom). How to engage the class in home activities remains, thus, an open problem. 
Another advantage of using the Geogebra applet at home revealed to be the possibility, for the tutor, to resort to it in class as a shared tool, which everybody knows and is able to use. Moreover, the in class activity with Socrative allowed the tutors to identify the weaknesses in the students' knowledge and to intervene timely and effectively. Socrative has been confirmed to be a tool for effective, in the moment, formative assessment.

\section{References}

Clark, M., \& Lovric, M. (2008). Suggestion for a theoretical model for secondary-tertiary transition in mathematics. Mathematics Education Research Journal, 20(2), 25-37.

Gómez-Chacón, I. M., Griese, B., Rösken-Winter, B., \& Gónzalez-Guillén, C. (2015, February). Engineering students in Spain and Germany-varying and uniform learning strategies. In CERME 9-Ninth Congress of the European Society for Research in Mathematics Education (pp. 2117-2123).

Gueudet, G. (2008). Investigating the secondary-tertiary transition. Educational studies in mathematics, 67(3), 237-254.

Habre, S. (2009). Multiple representations and the understanding of Taylor polynomials. PRIMUS, 19(5), 417-432.

Smith, T. I., Thompson, J. R., \& Mountcastle, D. B. (2013). Student understanding of Taylor series expansions in statistical mechanics. Physical Review Special Topics-Physics Education Research, 9(2), 020110.

Tall, D. (2004). Building theories: The three worlds of mathematics. For the learning of mathematics, 24(1), 29-32. 